\title{
Study of the Flow Induced by a Sliding Discharge
}

\author{
R. Sosa \\ University of Buenos Aires and CONICET \\ Av. Paseo Colón 850, C1063ACV \\ Buenos Aires, Argentina \\ E. Arnaud \\ INRIA Rhône-Alpes \\ 655, avenue de l'Europe 38330 \\ Montbonnot, France \\ E. Memin \\ INRIA, IRISA, \\ University of Rennes 1 , \\ 35042 Rennes Cedex, France \\ and G. Artana \\ University of Buenos Aires and CONICET. \\ Av. Paseo Colón 850, C1063ACV \\ Buenos Aires, Argentina
}

\begin{abstract}
In this work, we report on electrical and fluid-dynamics studies concerning the flow induced by a sliding discharge (SD). This kind of discharge was created with a three electrode system configuration: one excited with ac and the others with a dc negative voltage. The $S D$ was activated on a quiescent fluid at atmospheric pressure. The flow field induced by the $S D$ was analysed by measurements undertaken with Pitot probes and Schlieren Image Velocimetry. Under the conditions of our experiments two "jet flows", that blown towards the interelectrode space, were induced from the air exposed electrodes. As a consequence of the mutual interaction of these two flows and of the magnitude of each flow, a resulting plume like planar jet of adjustable direction (0$180^{\circ}$ ) could be formed. A robust control of the axis direction of the plume could be achieved by modifying the ac voltage value.
\end{abstract}

Index Terms — surface discharges, plasma devices, flow control.

\section{INTRODUCTION}

THE control of subsonic externals flows by means of plasma actuators have been receiving special attention in the last years [1]. In these plasma actuators a non-thermal surface plasma is produced.

In these devices the flowing air close to the surface of the body is ionized and as a consequence of the elastic collisions between the migrating charged particles and the neutral species of the gas, the neutrals particles increase their momentum giving rise to an 'electric wind' that takes place in the close vicinity of the body surface.

Other mechanisms like alterations of the physical properties of the gas (density, viscosity, etc), may eventually contribute

Manuscript received on 3 April 2008, in final form 17 June 2008. to the electromechanical coupling discharge-airflow [2]-[3].

The plasma actuators are technologically attractive because of their simplicity (they have no moving parts) and their very short response time (typically in the order of tens of milliseconds, see for instance [1]).

Plasma actuators can be classified in two large groups considering the number of electrodes that they use. A first group comprises devices employing two electrodes arrangements. They can consist on electrodes separated by a dielectric barrier, usually known as dielectric barrier discharge $(D B D)$ actuators, or on devices with two bare electrodes flush mounted on the body surface (BED).

The $D B D$ actuators use periodically excited electrodes and the dielectric barrier interposed between them plays an important role in the stabilization of the discharge. Typically, this plasma actuator is composed of two plane electrodes 
flush-mounted on both sides of a dielectric plate. A large number of aerodynamic studies of flows actuated with $D B D$ have been carried out (see for instance [4-7]).

The BED may produce distinctive gaseous discharges depending on the applied potential and the flow conditions [8], these are: unipolar coronas discharge (UC), bipolar coronas $(B C)$ and plasma sheet discharge $(P S)$. In any case the electrodes excitation can be produced either with dc or ac voltage supply. Regardless the voltage supply feature (dc or AC) the produced discharges may present current pulses (i.e. streamers are present in positive coronas and PS and Trichel pulses appear in negatives coronas) superposed to DC currents.

Usual UC are characterized by one active electrode surrounded by an ionization region where free charges are created and low field regions where charged particles drift and react in their movement towards the passive electrode [9-10]. In bipolar coronas both electrodes are active during the discharge but still the drift region persists.

On the contrary, in PS discharges the dielectric surface of the interelectrode space appears covered by a thin film of ionized air or plasma sheet. The plasma sheet current is composed by a dc component and a large number of peaks with frequencies of the order of some $\mathrm{kHz}$ that have been associated to cathode directed streamers [11]. The streamers presence in the inter-electrode gap is mainly the responsible of the luminous aspect of this region. The aerodynamic performances of $B E D$ plasma sheet actuators have been reported in previous works for different flow conditions [1218]. The main drawback of $B E D$ plasma actuators is that the electrical discharge becomes unstable under certain atmospheric conditions. For instance, corona-to-arc transition have been detected in some cases when the relative air humidity exceeded a threshold of about 55\% [19].

More recently [20]-[21], a new group of plasma actuators consisting in three electrodes arrangements has been developed (TED). Figure 1 shows the arrangement where two electrodes are flush mounted on the air exposed surface (upper electrodes (1) and (3)), and a third one is placed on the opposite side of the insulating surface facing the inter electrode space (lower electrode, (2)). If the electrode (3) and the electrode (2) are grounded biased while the other upper electrode (1) is at an ac voltage (even applying the maximum VPP equal to $25 \mathrm{kV}$ ) always a typical single $D B D$ is established between electrodes (1) and (2), as is showed in Figure 1a.

However, when the electrode (1) is at an ac voltage and a high enough negative dc voltage is applied to electrodes (2) and (3), an ionization process occupying the whole interelectrode space, as in the case of PS discharges, appears (see Figure 1b). This last discharge is know as: sliding discharge (SD) [22].

Finally if electrodes (2) and (3) are connected to a positive DC voltage the discharge established is not clearly a sliding discharge. Indeed, there is no luminous plasma sheet as illustrated in Figure 1b. This discharge looks like a single $D B D$, as shown in Figure 1a.
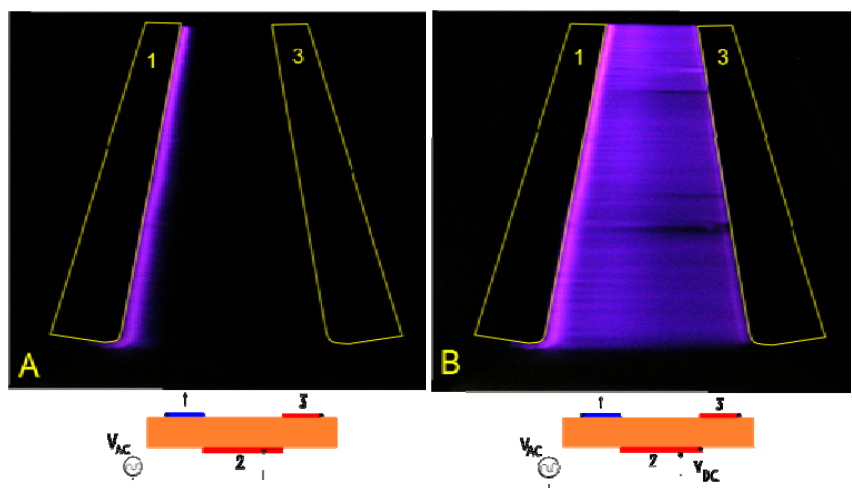

Figure 1. Different kinds of discharges produced by a three electrodes device (TED). The pictures are top viewed in perspective. a) Classical $D B D$ discharge, $V_{P P}=20 \mathrm{kV}$ (peak to peak value of the $A C$ voltage, sin waveform $f=9.5 \mathrm{kHz})$, b) Sliding discharge $(S D), V_{P P}=20 \mathrm{kV}, V_{D C}=$ $15 \mathrm{kV}$.

The electrical characteristics and aerodynamic performance of the sliding discharge $(S D)$ remain still rather unknown [20][21-23]. However this kind of discharge presents as main advantages less scaling problems in large bodies (large plasma sheets may be produced) and large stability (no arc transition at moderate DC potential).

We proposed here to study the flow induced by a sliding discharge (TED plasma actuator with electrodes (2) and (3) connected to a negative dc potential) when it is applied on quiescent air in order to optimize this use as an active flow control method. The aerodynamic effects were evaluated with Pitot measurements close to the air exposed electrodes. Flow visualisations were undertaken with a Schlieren technique using tracers not susceptible of charging problems. Quantification of the induced velocity fields was studied from these images by the use of Schlieren Image Velocimetry technique $(S I V)[24,25]$.

\section{EXPERIMENTAL SET-UP}

A scheme of the experimental set up is shown in Figure 2. The electrode arrangement of our experiment consists in two flat aluminium foils (electrodes (1) and (3)) flush mounted on the surface of a PMMA flat plate $(450 \mathrm{~mm} \times 300 \mathrm{~mm}$, thickness of $4 \mathrm{~mm}$ ) and a third one disposed at the opposite side (electrode (2)). The electrodes dimensions are $50 \mu \mathrm{m}$ thickness, length $400 \mathrm{~mm}$ (in the $z$ direction, see Figure 2) and different widths (5 mm for electrodes (1) and (3) and $30 \mathrm{~mm}$ for the electrode (2)). The inter-electrode gap between electrodes (1) and (3) was $30 \mathrm{~mm}$.

A dc power supply (-20 kV, $10 \mathrm{~mA})$ biased electrodes (2) and (3). An ac sinusoidal voltage excitation (frequency: $f=$ $9.5 \mathrm{kHz}$, zero mean value; peak to peak voltage: $V_{P P}$ from 0 to $25 \mathrm{kV}$ ) was applied to electrode (1). The ac power supply consisted in a function generator coupled to an audio amplifier (of 150 watt) that fed a high voltage transformer coil [26].

The electric current flowing in the system, $I(t)$, was measured with a shunt resistance, $R=1 \mathrm{k} \Omega$, connected to an 
oscilloscope with $60 \mathrm{MHz}$ of analogical bandwidth and $1 \mathrm{Gs} / \mathrm{s}$ of sampling rate (Figure 2). The AC voltage applied to electrode $1, V(t)$, was measured with a HV probe $(1000 \times / 3.0$ $\mathrm{pF} / 100 \mathrm{M} \Omega)$.

The PMMA flat plate, with the TED-SD plasma actuator, was placed horizontally in the test section of a low speed wind tunnel with a span length, in the $z$ direction, of $450 \mathrm{~mm}$ (the same length has the PMMA flat plate that supports the electrodes).

Two pressure probes (Pitot tubes: $\mathrm{P}_{1}$ and $\mathrm{P}_{3}$ in Figure 2) were mounted on the dielectric surface, placed at $3 \mathrm{~mm}$ in front of electrode $1(x=3 \mathrm{~mm}, \mathrm{y}=0.48 \mathrm{~mm})$ and of electrode $3(x=27 \mathrm{~mm}, \mathrm{y}=0.48 \mathrm{~mm})$ respectively. These probes were made of electrically insulating glass tubes (internal diameter: $I D=0.97 \mathrm{~mm}$, outer diameter $O D=1.3 \mathrm{~mm}$ ) and were connected by mean of tygon tubes to a differential pressure transducer of variable reluctance type ( 0 to $55 \mathrm{~Pa}$, accuracy of $0.25 \%$ full scale). The reference pressure value was measured at the test section but far away from the electrodes.

To visualize the induced ionic wind a Schlieren system disposed in a $Z$ configuration was used [27]. It comprised two spherical mirrors of $35 \mathrm{~cm}$ in diameter and the light (a tungsten-halogen automotive lamp was used as light source) was cut off with two razor blades, thus $2 \mathrm{D}$ density gradients could be detected. The parallel light rays traversed the test section of the low speed wind tunnel through circular windows (diameter $35 \mathrm{~cm}$ ) of optical quality disposed in coincidence with the parallel light beam. The images were recorded on a monochromatic digital image camera of 12 bits that enabled fast frame acquisition (up to 200 frame/s).

The Schlieren technique is able to detect changes in index refraction associated to density gradients in a fluid media. In general the small changes in density associated to the heating produced by the discharge enable to obtain images with sufficient contrast. In this context the "tracers" are the same fluid particles (the studied ones) and therefore there is not need to introduce some "external" particles (like smoke particles in the PIV systems).

However in order to improve the image contrast and gain insight of the induced flow in regions were the heated gas was not present, in some cases small quantities of Helium were injected vertically upwards from the surface through two small tubes $(I D=2.5 \mathrm{~mm})$ at 2.5 centimetres away from the interelectrode space.. The velocity of both He jets was about $0.5 \mathrm{~m} / \mathrm{s}$.

We may assume that the He injection does not significantly change the $S D$ characteristics because the values of the electrical magnitudes that we have monitored in our experiments were not affected by the He presence.

The Schlieren images were analysed with a recently developed method for motion estimation of the velocity field: Schlieren Image Velocimetry (SIV) [24, 25].

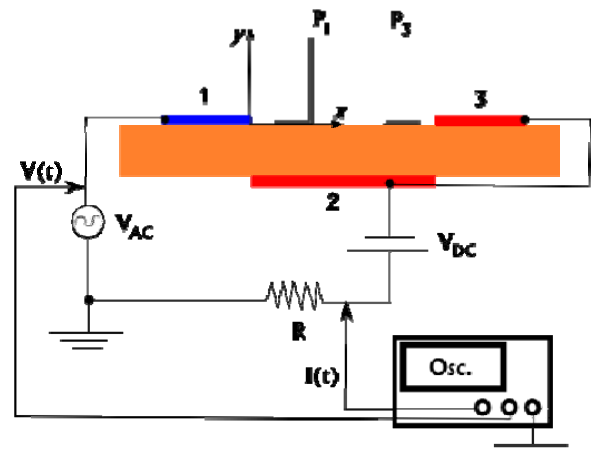

Figure 2. Experimental set-up.

\section{EXPERIMENTAL RESULtS}

\subsection{ELECTRICAL RESULTS}

The electric current $I(t)$ and the AC applied voltage, $V(t)$, of individual signals were recorded. Also by setting the oscilloscope in the average acquisition mode we have acquired the mean of these signals over 128 samples:

$$
\begin{aligned}
& I_{m}(t)=\frac{1}{128} \sum_{1}^{128} I(t) \\
& V_{m}(t)=\frac{1}{128} \sum_{1}^{128} V(t)
\end{aligned}
$$

The capacitive current, $I_{C}(t)$, was calculated as

$$
I_{C}(t)=C \frac{d V(t)}{d t}
$$

being $C$ the circuit capacitance. This was determined experimentally by applying a voltage $V_{0}(t)$ so low to inhibit any gas discharge. Measuring the corresponding $I_{0}(t), C$ could be calculated as [28]

$$
C=\frac{I_{0}(t)}{d V_{0}(t) / d t}
$$

Figure 3 shows typical signals of $I(t), I_{m}(t), I_{C}(t)$ and $V(t)$, the discharge current, the mean signal discharge current, the capacitive current and the ac voltage respectively.

When the TED is operated in a single $D B D$ mode (electrodes (2) and (3) grounded) we can observe on Figure 3a that in both parts of the ac cycle (positive and negative) a gaseous discharge occurs (i.e. $I(t)$ differs from $I_{C}(t)$ ). These results are in coincidence with many others already published by different authors [1].

When the TED actuator is operated in $S D$ mode (electrodes (2) and (3) at negative dc potential) during one fraction of the the positive ac cycle the voltage difference between electrodes enables the ignition of the sliding discharge. The total current differs substantially from the capacitive current (and from the single $D B D$ current, see Figure $3 \mathrm{a}$ ). It was determined that the 
higher current peaks appearing during the $S D$ formation (see the positive cycle in Figure $3 \mathrm{~b}$ ) were positive streamers (cathode directed) bridging the interelectrode gap [23].

During the negative part of the ac cycle no streamer traverses the interelectrode space but the current is not just capacitive and during this part of the cycle the discharge current does not substantially differ from the one observed in the negative phase of the single $D B D$ mode.

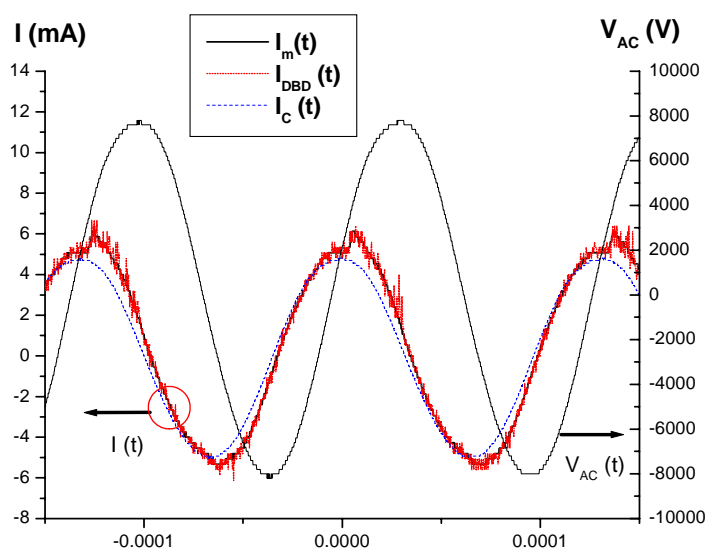

$t(s)$

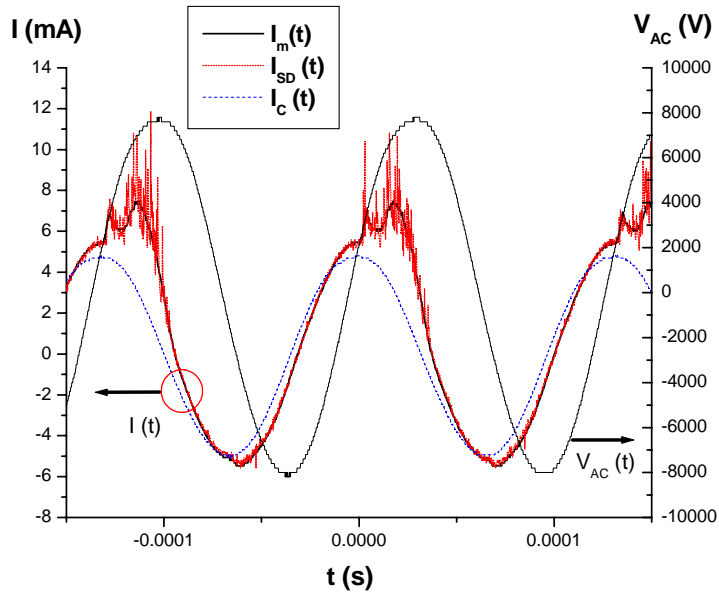

Figure 3. a) (upper) $I_{D B D}(t), I_{m}(t), I_{C}(t), V(t)$ for a $D B D\left(V_{D C}=0 \mathrm{kV}, \quad V_{P P}\right.$ $=16 \mathrm{kV})$; b) (lower): $I_{S D}(t), I_{m}(t), I_{C}(t), V(t)$ for a $S D\left(V_{D C}=-16 \mathrm{kV}, V_{P P}=\right.$ $16 \mathrm{kV})$.

In order to characterize the electric behavior of $S D$ the average current and the average power were analysed. Strictly these values must be determined using the $I(t)$ and $V(t)$ signals but only small errors were produced deriving them from the mean signals $\left(I_{m}\right.$ and $\left.V_{m}\right)$ and so we have decided to simplify our analysis and just consider the mean signals. The average current $\left(I_{a}\right)$ and power $\left(P_{a}\right)$ were calculated in consequence as:

$$
\begin{aligned}
& I_{a}=\frac{1}{T} \int_{0}^{T} I_{m}(t) d t \\
& P_{a}=\frac{1}{T} \int_{0}^{T} I_{m}(t) \cdot V_{m}(t) d t
\end{aligned}
$$

With $T$ the ac voltage period. Figure 4 shows the behavior of $I_{a}$ vs. $V_{P P}$ (peak to peak value of the ac voltage) for different $V_{D C}$ values.

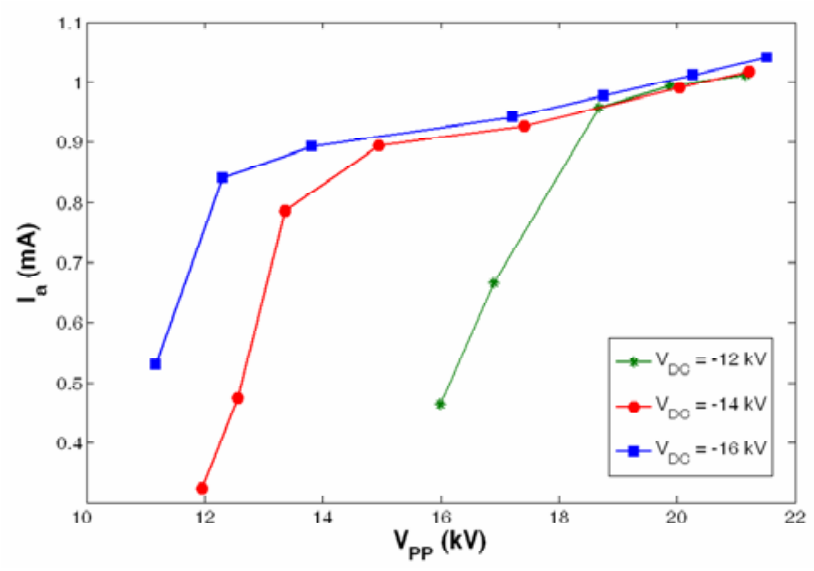

Figure 4. $l_{a}$ vs $V_{P P}$.

In all these curves it is possible to observe that initially there was an important increase of average current with the acvoltage. This behavior however changed at a given value of $V_{P P}$ (that depended on $V_{D C}$ value) and from this threshold value $I_{a}$ resulted in an almost linear function of $V_{P P}$ with a more moderate slope. The slope and current values of this "linear moderate regime" for the different tests were almost independent of the applied dc voltage values. However, the threshold voltage at which the $I_{a}$ VS $V_{P P}$ curve change its behavior occurred at lower $V_{P P}$ values when the negative voltage $\left(V_{D C}\right)$ increase. For values of $V_{P P} \geq 18 \mathrm{kV}$ the observed $I_{a}$ values resulted almost independent of the $V_{D C}$ values.

In Figure 5 we represent the average power as a function of $V_{P P}$. For the cases here analysed at $V_{P P}$ values higher than 18 $\mathrm{kV}$, the differences in power consumption for the different $V_{D C}$ values remained almost constant. This behaviour corresponded to the actuator operation in the moderate linear regime of $I_{a}$ vs $V_{P P}$.

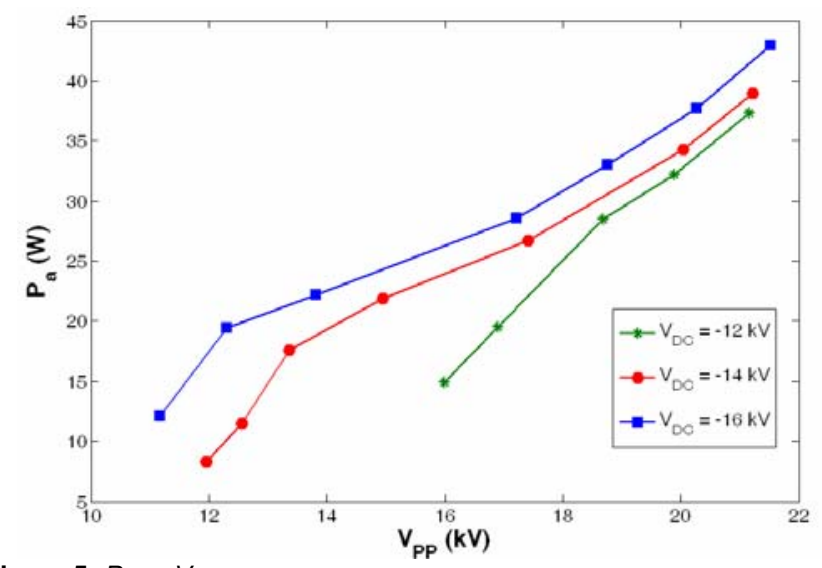

Figure 5. $P_{a}$ VS $V_{P P}$.

At present, the physical mechanisms involved in the two different regimes with different slope appearing in the curve $I_{a}$ vs. $V_{P P}$ (and consequently in the curve $P_{a}$ vs. $V_{P P}$ ) are under 
study in our laboratory. The "linear moderate regime" might be probably related to external limitations of the electric circuit associated to a limiting value of the power $(20 \mathrm{~W})$ of our dc power source. However, from our studies still we can not conclude that the sliding discharge has not a self limiting current mechanism.

\subsection{FLUID DYNAMICS RESULTS}

The induced flow velocities registered with the Pitots $P_{1}$ and $P_{3}$ (named $U_{1}$ and $U_{3}$ respectively) can be observed on Figure 6 . These measurements revealed that two distinctive flows directed towards the inter-electrode space were induced from both electrodes. In front of the electrode (1) (always acting as anode respect to electrode (3)) the module of the velocity of the induced wind increased almost linearly with the $V_{P P}$ value. This velocity did not show a clear dependency with respect to the $V_{D C}$ values, and curves for different $V_{D C}$ values result almost coincident. However the flow induced from this electrode shows a similar dependency with $V_{P P}$ as the one observed in single $D B D$ mode operation [1].

On the other hand in face of the electrode (3) (always acting as cathode respect to electrode (1)) the induced wind showed an almost constant value or decreased slightly when increasing $V_{P P}$. Concerning the influence of $V_{D C}$ on these flows we observed that for a given value of $V_{P P}$ the velocity at $P_{1}$ resulted almost null for low values of $V_{D C}$ but increased substantially for higher $V_{D C}$ values. These results seem to indicate that the flow originated from this electrode could be associated to an activation of this electrode in a similar way as occurs in bipolar coronas.

In terms of average power the velocity $U_{1}$ (Figure 7) exhibited a constant slope in correspondence with the linear moderate regime. As can be observed the actuation was more effective to induce flow in this regime and it could not be observed a saturation of velocity with the power. However it should be mentioned that we could not undertake our tests at very large values of power as a consequence of limitations imposed by the maximum power that could be delivered by our experimental setup.

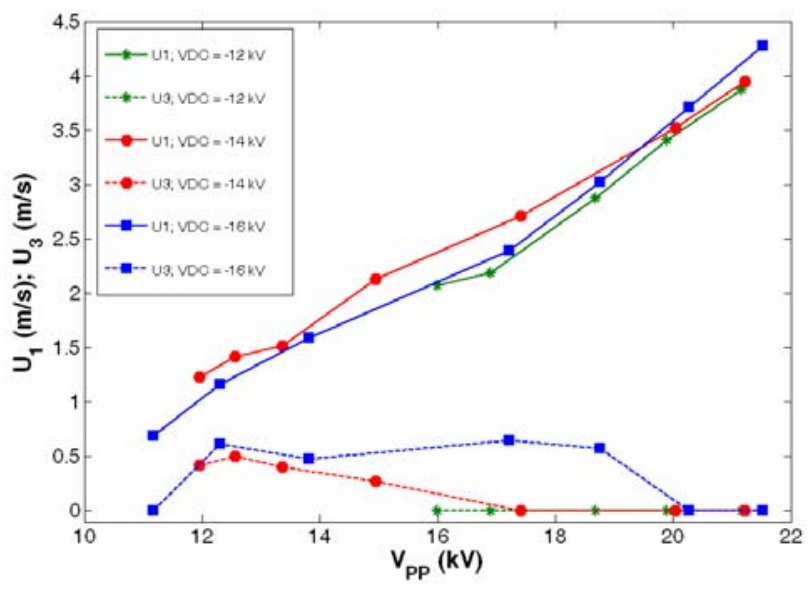

Figure 6. $U_{1}$ vs $V_{P P}$ and $U_{3}$ Vs $V_{P P}$

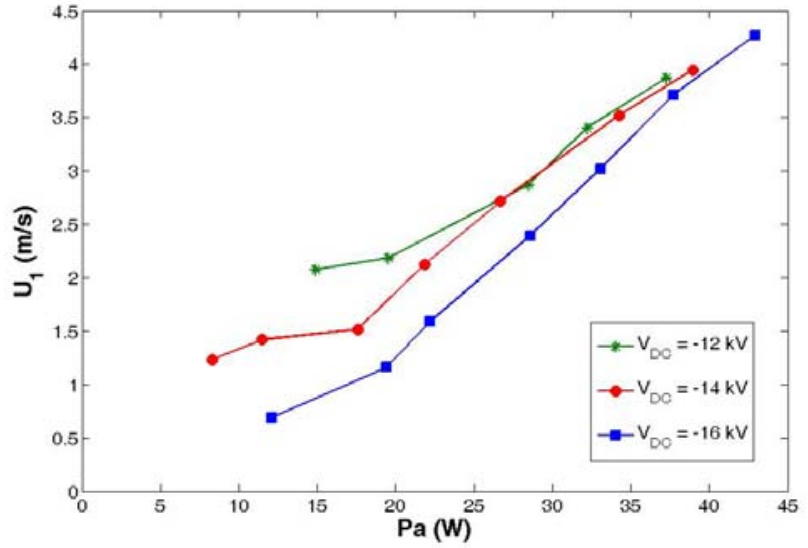

Figure 7. $U_{1}$ vs $P_{a}$.

In order to better understand the resulting flow produced by these two opposite induced flows, a set of Schlieren images were processed to obtain the associated velocity fields. Figures 8, 9 and 10 shows the instantaneous flow visualization produced by the Schlieren system and the associated time averaged velocities fields over 30 instantaneous velocities fields.

Visualizations and SIV results show that the discharge-fluid electromechanical coupling is relative high in all cases as the region of neutral gas affected by the $S D$ is relative wide and the action spreads in a relative large region.

The analysis of these results shows that under the conditions of our experiments two "jet flows" were induced from the air exposed electrodes. These ionic wind jets blow towards the inter-electrode space but their flow configuration are not exactly symmetric. As a consequence of the mutual interaction of these two flows and of the magnitude of each one, a resulting plume like planar jet of adjustable direction $\left(0-180^{\circ}\right)$ could be formed.

It is worth noting that similar jet vectoring could be achieved by pointing two single $D B D$ plasma actuator at each other. Recently [29] it has bee found that, varying the amplitude of the high voltage applied to each $D B D$ actuator, the jet can be inclined up to around $20^{\circ}$ without any loss in power. Other researches, working with these $D B D$ plasma synthetic jets [30] have found that the angle of the jet could be controlled spanning 180 degrees through small changes in the voltage applied to the electrodes.

For high values of $V_{P P}$ (Figure 8) the jet from the electrode 1 (at right), the one commonly observed in the single $D B D$ mode operation, prevailed from the one of electrode 3 (at left) and the global plume could be directed to the right of the image as occurs in single $D B D$ mode operation.

Note that velocity measured by Pitot probes correspond to regions in close proximities to the electrodes and to the dielectric surface were maximum velocities of the induced flow were attained. In these regions, the intensity of schlieren images saturated and changes between two successive images were rather difficult to be observed. Far from these regions punctual velocities detected by Pitot probes and SIV agreed satisfactorily. 

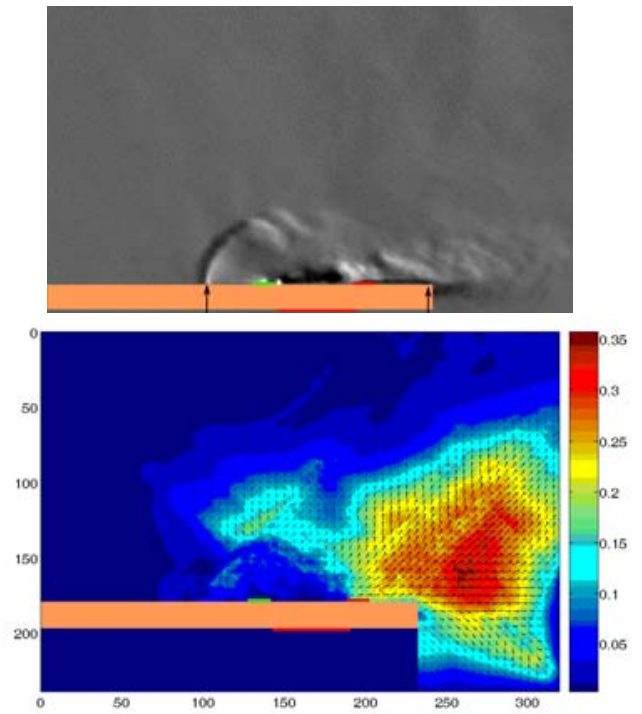

Figure 8. a) (upper) Schlieren image (arrows indicate the He injections points); b) (lower) Time averaged velocity field obtained by (SIV) in $\mathrm{m} / \mathrm{s}, V_{D C}=-16 \mathrm{kV}, V_{P P}=21.8 \mathrm{kV}, l_{a}=0.94 \mathrm{~mA}, P_{a}=44.31 \mathrm{~W}-$ Electrode arrangement agrees with the one of Figure 2.

When the $V_{P P}$ was decreased (for the same $V_{D C}$ value considered) the induced flow from electrode 1 was decreased while the induced flow velocity from electrode 3 remained almost constant. Under these conditions the flows produced from both electrodes equilibrate in such a way that a global plume almost perpendicular to the dielectric plate was observed (see Figure 9).

Finally at reduced values of $V_{P P}$ (Figure 10) the flows from the electrodes interacted in such a way that they produce a global "plume" directed to the left of the image. The jet produced from electrode 1 does not prevail from the one produced from electrode 3 and the plume is directed in the contrary sense of single $D B D$ operation.

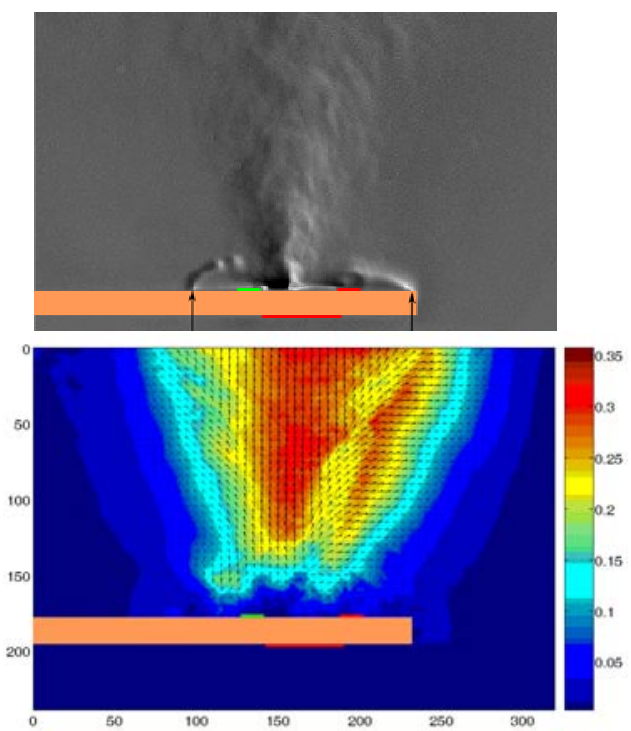

Figure 9. a) (upper) Schlieren image (arrows indicate the He injections points); b) (Lower) Time averaged velocity field obtained by (SIV) in $\mathrm{m} / \mathrm{s}, V_{D C}=-16 \mathrm{kV}, V_{P P}=18.4 \mathrm{kV}, I_{a}=0.82 \mathrm{~mA}, P_{a}=30.46 \mathrm{~W}$ Electrode arrangement agrees with the one of Figure 2.
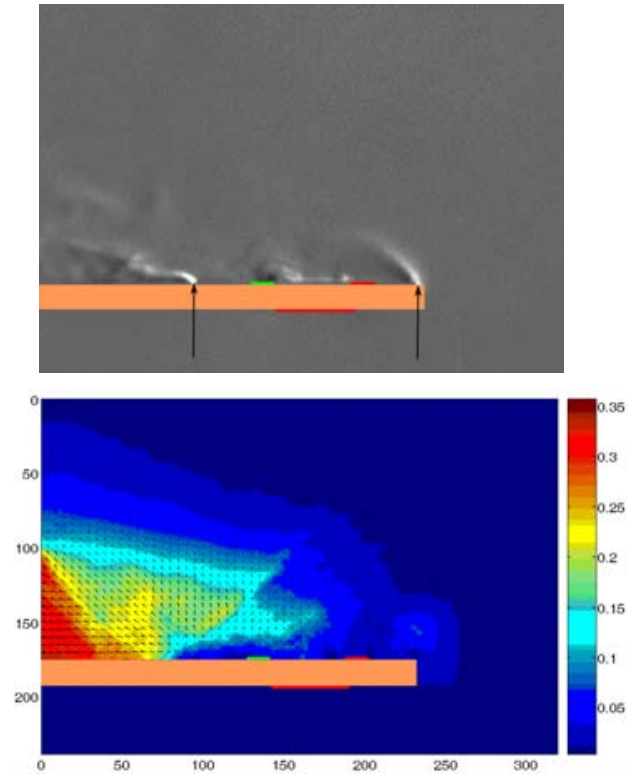

Figure 10. a) (upper) Schlieren image (arrows indicate the $\mathrm{He}$ injections points); b) (lower) Time averaged velocity field obtained by $(S I V)$ in $\mathrm{m} / \mathrm{s}, V_{D C}=-16 \mathrm{kV}, V_{P P}=12.5 \mathrm{kV}, I_{a}=0.60 \mathrm{~mA}, P_{a}=15.84 \mathrm{~W}$ .Electrode arrangement agrees with the one of Figure 2.

\section{Conclusion}

The flow induced by the $S D$ on the electrodes proximity was analyzed by means of Pitot probes and flow field obtained with SIV. At relative high values of the DC voltage both electrodes become active. Under this condition two induced jet flows, with origin at the electrodes proximity, resulted directed towards the interelectrode space.

The global induced flow direction was strongly dependent on the applied ac voltage. By varying the ac voltage value the global plume like flow direction could span angles almost lying in the range $0-180^{\circ}$ referred to the dielectric surface.

This work probed that the $S D$ is a promising tool in flow control areas because of their capability to induce a variable direction flow with similar velocities than other plasma actuators with fixed direction of action. The large extension of the ionization region (covering all the inter electrode space) of the $S D$ may produce also substantial alterations of the physical properties of the gas (density, viscosity, etc) in the regions very close to the flow boundaries. Even though this effect could further improve the electromechanical coupling air discharge in different situation this was not explored in this work.

\section{ACKNOWLEDGMENT}

This work was supported by The Argentine government grants UBACYT 0105/I024.

\section{REFERENCES}

[1] E. Moreau, "Airflow control by non-thermal plasma actuators", J. Phys. D: Appl. Phys., Vol. 39, pp. 1-32, 2006. 
[2] R. Sosa, "Mecanismos de Acople en actuadores EHD", PhD Theses, University of Buenos Aires, Faculty of Engineering, October 2007 (in Spanish)

[3] Scherbakov A. et al., "Drag reduction by ac streamer corona discharges along a wing-like profile plate", AIAA Paper No 2000-2670, 2000.

[4] J. R. Roth and D. Xin, "Optimization of the aerodynamic plasma actuator as an electrohydrodynamic (EHD) electrical device", AIAA Paper No 2006-1203, 2006.

[5] T. Corke and M. Post, "Overview of plasma flow control: concepts, optimization, and applications”, AIAA Paper No 2005-563, 2005.

[6] S. P. Wilkinson, "Investigation of an oscillating surface plasma for turbulent drag reduction", AIAA Paper No 2003-1023, 2003.

[7] M. Forte, J. Jolibois, J. Pons, E. Moreau, G. Touchard and M. Cazalens, "Optimization of a dielectric barrier discharge actuator by stationary and non-stationary measurements of the induced flow velocity: application to airflow control", Exp. Fluids, Vol 43 Nº, pp. 917-28, 2007.

[8] E. Moreau, L. Leger and G. Touchard., "Effect of a DC surface nonthermal plasma on a flat plate boundary layer for airflow velocity up to 25m/s", J. Electrostatics, 64, pp. 215-25, 2006.

[9] G. Colver and S. El-Khabiry, "Modelling of DC corona discharge along an electrically conductive flat plate with gas flow", IEEE Trans. Ind. Appl. 35, pp. 387-94, 1999.

[10] C. Noger, J. S. Chang and G. Touchard, "Active controls of electrohydrodynamically induced secondary flow in corona discharge reactor", Proc. 2nd Int. Symp. on Plasma Technology and Polution Control, Bahia, Brazil pp. 136-41, 1997.

[11] R. Sosa, G. Artana, D. Grondona, H. Kelly, A. Márquez and F. Minotti, "Discharge characteristics of plasma sheet actuators", J. Phys. D: Appl. Phys. 40 No 3, pp. 663-673, 2007.

[12] G. Artana, J. D'Adamo, L. Leger, E. Moreau and G. Touchard, "Flow control with electrohydrodynamic actuators", AIAA J. 40, pp. 1773-9, 2002 .

[13] G. Artana, R. Sosa, E. Moreau and G. Touchard, "Control of the near wake flow around a circular cylinder with electrohydrodynamic actuators", Exp. Fluids 36, pp. 580-8, 2003.

[14] R. Sosa and G. Artana, "Steady control of laminar separation over airfoils with plasma sheet actuators", J. Electrostat. 64, pp. 604-10, 2006.

[15] R. Sosa, E. Moreau, G. Touchard and G. Artana, "Stall control at high angle of attack with periodically excited EHD actuators", AIAA Paper No 2004-2738, 2004.

[16] R. Sosa, G. Artana, E. Moreau and G. Touchard, "Stall control at high angle of attack with plasma sheet actuators", Exp.Fluids, Vol. 42, No 1, pp. 143-167, 2007.

[17] P. Magnier, D. Hong, A. Leroy-Chesneau, J. Pouvesle and J. Hureau, "A DC corona discharge on a flat plate to induce air movement", Journal of Electrostatics, Volume 65, Issues 10-11, pp. 655-659, 2007.

[18] P. Magnier, D. Hong, A. Leroy-Chesneau, J. Bauchire and J. Hureau, "Control of separated flows with the ionic wind generated by a DC corona discharge", Exp Fluids 42, pp. 815-825, 2007.

[19] L. Léger, E. Moreau and G. Touchard, "Effect of a DC corona electrical discharge on the airflow along a flat plate", IEEE Trans. Indust. Appl. 38 , pp. $1478-85,2002$.

[20] C. Louste, G. Artana, E. Moreau and G. Touchard, "Sliding discharge in air at atmospheric pressure: electrical properties" J. of Electrostatic, Vol. 63, pp. 615-620, 2005

[21] E. Moreau, C. Loustea and G. Touchard, "Electric wind induced by sliding discharge in air at atmospheric pressure", J. of Electrostatic, Vol. 66, pp. 107-14, 2008.

[22] A. Lagarkov and I. Rutkevich, "Ionization Waves in Electrical Breakdown of Gases", Springer-Verlag, NY, pp. 195-207, 1993.

[23] R. Sosa, H. Kelly, D. Grondona, A. Márquez, V. Lago and G. Artana, "Electrical and plasma characteristics of a quasi-steady sliding discharge", J. Phys. D: Appl. Phys., Vol 41035202 (8pp), 2008.

[24] E. Arnaud, E. Memin, R. Sosa and G. Artana, "A Fluid Motion Estimator for Schlieren Image Velocimetry", Lecture Notes in Computer Science, 3951 / 2006, pp. 198 - 210, 2006.

[25] R. Sosa, E. Arnaud, E. Memin and G. Artana, "Schlieren Image Velocimetry applied to EHD flows", Proc. of the Int. Symposium on Electrohydrodonamics (ISEHD), pp. 331-334, 2006.

[26] M. Yadav, X. Dai and J. R. Roth, "Measurement of the Boundary Layer Velocity Induced by Paraelectric Plasma Actuators using an Inexpensive High Voltage Power Supply", Paper 3P-38, Proc. 31st IEEE Int.
Conference on Plasma Science, pp. 244, IEEE Catalog No. 04CH37537, ISBN 0-7803-8334-6, 2004.

[27] G. S. Settles, "Schlieren and Shadowgraph techniques”, Springer , NY, 2001.

[28] M. Petit, N. Jidenko, A. Goldman, M. Goldman and J. P. Borra, "Electrical characterization of gas discharges using a numerical treatment. Application to dielectric barrier discharges", Review of Scientific Instruments, volume 73, number 7, pp. 2705-2712, 2002.

[29] N. Bénard, J. Jolibois, E. Moreau, R. Sosa, G. Artana and G. Touchard, "Aerodynamic plasma actuators: A directional micro-jet device", Thin Solid Films, Vol. 516, Issue 19, pp. 6660-67, 2007.

[30] C. Porter, A. Abbas, K. Cohen, T. McLaughlin, and C. L. Enloe, "Spatially Distributed Forcing and Jet Vectoring with a Dielectric Barrier Discharge Plasma Actuator", AIAA 2008-1374, 2008.

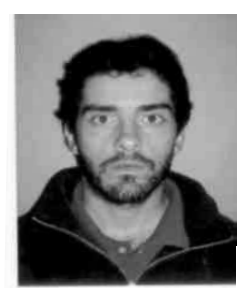

R. Sosa was born in Buenos Aires, Argentine in 1978. He received the M.S. and Ph.D. degrees in Engineering from Buenos Aires University, Buenos Aires, Argentina, in 2002 and 2007, respectively. Since 2000, he has been a researcher with the Fluidynamics Laboratory, Faculty of Engineering, University of Buenos Aires. His current research interests are plasma flow control and nonthermal high-pressure plasmas. Since 2008, he has been a researcher with CONICET.

E.. Arnaud presently not available

E.. Memin presently not available

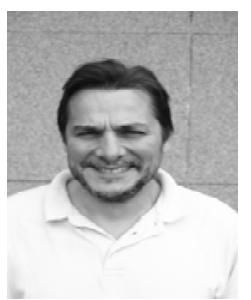

G. Artana was born at Buenos Aires in 1963. He obtained his degree of Enginner of the University of Buenos Aires in 1989 and in 1995 his $\mathrm{PhD}$ at the University of Poitiers. Since 1997 is a researcher of CONICET and at present is professor of the Department of Mechanical Engineering and head of the Fluidynamics Laboratory of the University of Buenos Aires. Research areas of interest are flow control and continuum electromechanics. 